\section{Periphere Analgetika auch bei starken Schmerzen gut wirksam}

\begin{abstract}
Hydrocodon und Oxycodon werden vor allem in den USA wahrscheinlich häufiger als nötig verordnet. Eine Vergleichsstudie untersuchte deshalb die analgetische Potenz von Opioid-Kombinationen gegen eine ParacetamolIbuprofen-Kombi in Notfallambulanzen.
\end{abstract}

$V^{2}$ ermutlich erhöht bereits eine nur kurzfristige Opioideinnahme das Risiko für Opiatmissbrauch und Abhängigkeit. Entsprechend kritisch müssen Daten aus den USA gewertet werden, denen zufolge knapp $20 \%$ aller Patienten die Notfallambulanz mit einem Opioidrezept verlassen. Dies erfolgt offenbar in der Annahme, dass Opioide stärker analgetisch wirksam seien als Nichtopioidanalgetika. Klinische Studien aus Notfallambulanzen zu dieser Fragestellung liegen allerdings kaum vor. Eine Notfallambulanzstudie verglich deshalb den Effekt von verschiedenen Opioid-Kom-

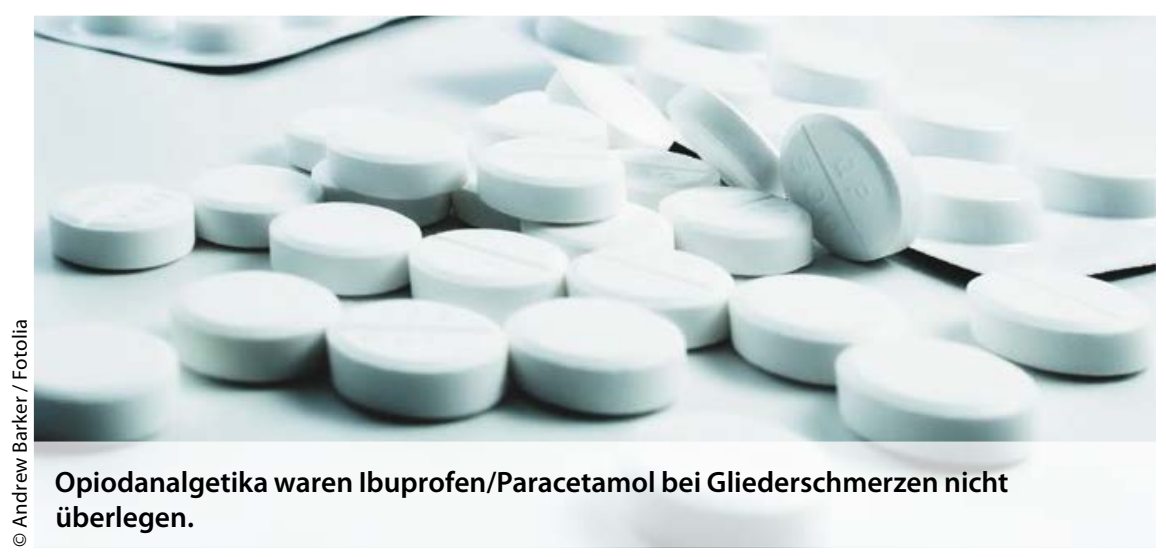

\section{Fibromyalgie-Schmerzen per Glutamatblockade lindern}

\section{Schmerzen bei Fibromyalgie beruhen möglicherweise auch auf erhöhten Konzentrationen des exzitatorischen Neurotransmitters Glutamat im zentra- len Nervensystem. Eine erste kleine Doppelblindstudie untersuchte deshalb die analgetischen Effekte des Glutamatblockers Memantin.}

\footnotetext{
chmerz ist eines der belastendsten

Symptome für Patienten mit Fibromyalgie. Eine Standardtherapie gibt es bisher nicht, eine pharmakologische In-
}

binationen gegen Ibuprofen plus Paracetamol bei Gliedmaßenverletzungen.

In die an zwei New Yorker Zentren durchgeführte Untersuchung wurden 416 erwachsene Patienten einbezogen, die randomisiert und doppelblind entweder $400 \mathrm{mg}$ Ibuprofen plus $1000 \mathrm{mg}$ Paracetamol oder drei Kombinationen rund $300 \mathrm{mg}$ Paracetamol plus ei(30 mg Codein, 5 mg Oxy(a) ls Bedarfsmedikation 5 mg Oxycodon zur Verfügung. Primäres Studienziel war die Gruppendifferenz beim analge-

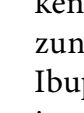
Ibuprofen-Paracetamol die Schmerzen innerhalb von zwei Stunden genauso effektiv wie Paracetamol-Kombinationen mit Hydro- oder Oxycodon beziehungsweise Codein in jeweils niedriger, aber in der Praxis gebräuchlicher Dosierung. Dr. Barbara Kreutzkamp

Chang AK et al. Effect of a single dose of oral opioid and nonopioid analgesics on acute extremity pain in the emergency department. JAMA 2017; 318:1661-7

weiterer Kandidat für die Schmerzbekämpfung bei Fibromyalgie ist Memantin. Die bereits seit rund 20 Jahren bekannte Substanz reduziert die neurotoxischen Effekte von Glutamat durch Bindung an den N-methyl-D-AspartatRezeptor, was sich unter anderem in der Behandlung von Morbus Parkinson und Alzheimer-Demenz positiv bemerkbar macht. Die Rationale für den Einsatz bei Fibromyalgie sind neuere Befunde, denen zufolge Glutamat auch an einer überschießenden Schmerzempfindung 